\title{
On rigidity of analytic black holes
}

\author{
Piotr T. Chruściel* \\ Département de Mathématiques \\ Faculté des Sciences \\ Parc de Grandmont \\ F37200 Tours, France
}

July 3, 2018

\begin{abstract}
We establish global extendibility (to the domain of outer communications) of locally defined isometries of appropriately regular analytic black holes. This allows us to fill a gap in the Hawking-Ellis proof of black-hole rigidity, for "non-degenerate" black-holes.
\end{abstract}

\section{Introduction}

According to Hawking and Ellis [11, Prop. 9.3.6], under appropriate conditions, which include analyticity of all the objects under consideration, the event horizon of a stationary, say electro-vacuum, black hole space-time $(M, g)$ is necessarily a Killing horizon. More precisely, the isometry group of $(M, g)$ should contain an $\mathbb{R}$ subgroup, the orbits of which are tangent to the black hole horizon. In order to substantiate their claim the authors of [11] first argue that for each $t$ the map defined as the translation by $t$ along the appropriately parameterized generators of the event horizon extends to an

*On leave of absence from the Institute of Mathematics, Polish Academy of Sciences, Warsaw. Supported in part by KBN grant \# 2 P301 105 007. E-mail: Chrusciel@UnivTours.fr 
isometry $\phi_{t}$ in a neighborhood of the event horizon. Next they assert that for all $t$ one can analytically continue $\phi_{t}$ to the whole space-time, to obtain a globally defined one parameter group of of isometries. This last claim is wrong, which" can be seen as follows: Let $\left(M, g_{a b}\right)$ be the extension of the exterior region of the Kerr space-time consisting of "two type I regions and two type II regions", as described in Section 5.6 of 111 (thus $\left(M, g_{a b}\right)$ consists of the four uppermost blocks of Figure 28, p. 165 in [11]). Let $\phi_{t}$ denote those isometries of $\left(M, g_{a b}\right)$ which are time-translations in an asymptotic region $M_{\text {ext }}$, and let $\left\langle\left\langle M_{\text {ext }}\right\rangle\right\rangle$ denote the domain of outer communication of $\left(M, g_{a b}\right)$ as determined by $M_{\text {ext }}$ ( $c f$. eq. (2.1) below; $M_{\text {ext }}$ corresponds to one of the blocks "I" of Figure 28 of [11]). Let $\Sigma$ be any asymptotically flat Cauchy surface of $\left(M, g_{a b}\right)$ (thus $\Sigma$ has two asymptotic regions), and let $\mathcal{E}$ be any embedded two-sided three-dimensional sub-manifold of $\operatorname{int} \mathcal{D}^{+}(\Sigma ; M) \backslash\left\langle\left\langle M_{\text {ext }}\right\rangle\right\rangle$, invariant under $\phi_{t}$. We shall moreover suppose that $M \backslash \overline{\mathcal{E}}$ is connected, and that $\mathcal{E}$ is not invariant under the $U(1)$ factor of the isometry group of $\left(M, g_{a b}\right)$. Let $\left(M_{a}, g_{a}\right), a=1,2$, be two copies of $M \backslash \overline{\mathcal{E}}$ with the metric induced from $g$. As $\mathcal{E}$ is two-sided, there exists an open neighborhood $\mathcal{O}$ of $\mathcal{E}$ such that $\mathcal{E}$ separates $\mathcal{O}$ into two disjoint open sets $\mathcal{O}_{a}, a=1$, 2, with $\overline{\mathcal{O}_{1}} \cap \overline{\mathcal{O}_{2}}=\overline{\mathcal{E}}, \mathcal{O}_{1} \cap \mathcal{O}_{2}=\emptyset$. Let $\psi_{a}$ denote the natural embedding of $\mathcal{O}_{a}$ into $M_{a}$. Let $M_{3}$ be the disjoint union of $M_{1}, M_{2}$ and $\mathcal{O}$, with the following identifications: a point $p \in \mathcal{O}_{a} \subset \mathcal{O}$ is identified with $\psi_{a}(p) \in M_{a}$. It is easily seen that $M_{3}$ so defined is a Hausdorff topological space.

We can equip $M_{3}$ with the obvious real analytic manifold structure and an obvious metric $g_{3}$ coming from $\left(M_{1}, g_{1}\right),\left(M_{2}, g_{2}\right)$ and $\left(\mathcal{O},\left.g\right|_{\mathcal{O}}\right)$. Note that $g_{3}$ is real analytic with respect to this structure. Let finally $\left(M_{4}, g_{4}\right)$ be any maximalf vacuum real analytic extension of $\left(M_{3}, g_{3}\right)$. Then $\left(M_{4}, g_{4}\right)$ is a maximal vacuum real analytic extension of $\left\langle\left\langle M_{\text {ext }}\right\rangle\right\rangle$ which clearly is not isometric to $(M, g)$.

The space-time $\left(M_{4}, g_{4}\right)$ satisfies all the hypotheses of [11]. The connected component of the identity of the group of isometries drops down from $\mathbb{R} \times U(1)$

\footnotetext{
${ }^{1}$ The construction that follows is a straightforward adaptation to the problem at hand of a construction in [8, Section 5].

${ }^{2}$ cf., e.g., [4. Appendix C] for a proof of existence of space-times maximal with respect to some property. It should be pointed out that there is an error in that proof, as the relation $\prec$ defined there is not a partial order. This is however easily corrected by adding the requirement that the isometry $\Phi$ considered there restricted to some fixed threedimensional hypersurface be the identity.
} 
(in the case of $\left.\left(M, g_{a b}\right)\right)$ to $\mathbb{R}$ (in the case of $\left(M_{4}, g_{4}\right)$ ), as all the orbits of the rotation group acting on $\left(M, g_{a b}\right)$ meeting $\mathcal{E}$ are incomplete in $\left(M_{4}, g_{4}\right)$.

Topological games put aside, the method of proof suggested in [11] of analytically extending $\phi_{t}$ faces the problem that $\phi_{t}$ might potentially be analytically extendible to a proper subset ${ }^{\beta}$ of the space-time only. One can nevertheless hope that the analyticity of the domain of outer communication and some further conditions, as e.g. global hyperbolicity thereof, allow one to extend the locally defined isometries at least to the whole domain of outer communications. The aim of this paper is to show that this is indeed the case. More precisely, we wish to show the following:

Theorem 1.1 Consider an analytic space-time $\left(M, g_{a b}\right)$ with a Killing vector field $X$ with complete orbits. Suppose that $M$ contains an asymptotically flat three-end $\Sigma_{\text {ext }}$ with time-like ADM four-momentum, and with $X(p)$ time-like for $p \in \Sigma_{\text {ext }}$. (Here asymptotic flatness is defined in the sense of eq. (2.9) with $\alpha>1 / 2$ and $k \geq 3$.) Let $\left\langle\left\langle M_{\text {ext }}\right\rangle\right\rangle$ denote the domain of outer communications associated with $\Sigma_{\text {ext }}$ as defined below, assume that $\left\langle\left\langle M_{\mathrm{ext}}\right\rangle\right\rangle$ is globally hyperbolic and simply connected. If there exists a Killing vector field $Y$, which is not a constant multiple of $X$, defined on an open subset $\mathcal{O}$ of $\left\langle\left\langle M_{\mathrm{ext}}\right\rangle\right\rangle$, then the isometry group of $\left\langle\left\langle M_{\mathrm{ext}}\right\rangle\right\rangle$ (with the metric obtained from $\left(M, g_{a b}\right)$ by restriction) contains $\mathbb{R} \times U(1)$.

\section{Remarks}

1. It should be noted that no field equations or energy inequalities are assumed.

2. Simple connectedness of the domain of outer communications necessarily holds when a positivity condition is imposed on the Einstein tensor of $g_{a b}$ 10. I

\footnotetext{
${ }^{3}$ In the physics literature there seem to be misconceptions about existence and uniqueness of analytic extensions of various objects. As a useful example the reader might wish to consider the (both real and complex) analytic function $f$ from, say, the open disc $D(1,1 / 2)$ of radius $1 / 2$ centered at 1 into $\mathbb{C}$, defined as the restriction of the principal branch of $\log z$. Then: 1) There exists no analytic extension of $f$ from $D(1,1 / 2)$ to $\mathbb{C}$. 2) There exists no unique maximal subset of $\mathbb{C}$ on which an analytic extension of $f$ is defined.

${ }^{4} c f$. also [9, 13, 11] for similar but weaker results. Note that in the stationary black hole context, under suitable hypotheses one can use Theorem 1.2 below to obtain completeness of orbits of $X$ in $\left\langle\left\langle M_{\text {ext }}\right\rangle\right\rangle$, and then use 9 to obtain simple-connectedness of $\left\langle\left\langle M_{\text {ext }}\right\rangle\right\rangle$.
} 
3. When a positivity condition is imposed on the Einstein tensor of $g_{a b}$, the hypothesis of time-likeness of the ADM momentum can be replaced by that of existence of an appropriately regular Cauchy surface in $\left(M, g_{a b}\right)$. See, e.g., [12] and references therein; $c f$. also [2] for a recent discussion.

4. It should be emphasized that no claims about isometries of $M \backslash\left\langle\left\langle M_{\text {ext }}\right\rangle\right\rangle$ (with the obvious metric) are made.

Theorem 1.1 allows one to give a corrected version of the rigidity theorem, the reader is referred to [7] for a precise statement together with a proof.

It seems of interest to remove the condition of completeness of the Killing orbits of $X$ above. Recall that completeness of those necessarily holds [5] in maximal globally hyperbolic, say vacuum, space-times under various conditions on the Cauchy data. (It was mentioned in [6] that the results of [5] generalize to the electro-vacuum case.) Those conditions are, however, somewhat unsatisfactory in the black hole context for the following reasons: recall that the existing theory of uniqueness of black holes gives only a classification of domains of outer communication $\left\langle\left\langle M_{\text {ext }}\right\rangle\right\rangle$. Thus in this context one would like to have results which do not make any hypotheses about the global properties of the complement of $\left\langle\left\langle M_{\text {ext }}\right\rangle\right\rangle$ in $M$. Moreover the hypotheses of those results of [5] which apply when degenerate Killing horizons are present require further justification. Here we wish to raise the question, whether or not it makes sense to talk about a stationary black hole spacetime for space-times for which the Killing orbits are not complete in the asymptotic region. We do not know an answer to that question. It is nevertheless tempting to decree that in "physically reasonable" stationary black hole space-times the orbits of the Killing vector field $X$ which is time-like in the asymptotically flat three-end $\Sigma_{\text {ext }}$ are complete through points in the asymptotic region $\Sigma_{\text {ext }}$. One would then like to be able to derive various desirable global properties of $\left\langle\left\langle M_{\text {ext }}\right\rangle\right\rangle$ using this assumption. Our second result in this paper is the proof that in globally hyperbolic domains of outer communication the orbits of those Killing vector fields which are time-like in $\Sigma_{\text {ext }}$ are complete "if and only if" they are sof for points $p \in \Sigma_{\text {ext }}$ (it should

\footnotetext{
${ }^{5}$ The quotation marks here are due to the fact that in our approach the asymptotic four-end $\left\langle\left\langle M_{\text {ext }}\right\rangle\right\rangle$ is not even defined when the orbits of $X$ through $\Sigma_{\text {ext }}$ are not complete. In that last case one could make sense of this sentence using Carter's definition of the domain of outer communication [3], involving Scri.
} 
be emphasized that, in contradistinction to [5], no maximality hypotheses are made and no field equations are assumed below; similarly no analyticity or simple connectedness conditions are made here):

Theorem 1.2 Consider a space-time $\left(M, g_{a b}\right)$ with a Killing vector field $X$ and suppose that $M$ contains an asymptotically flat three-end $\Sigma_{\text {ext }}$, with $X$ time-like in $\Sigma_{\text {ext }}$. (Here the metric is assumed to be twice differentiable, while asymptotic flatness is defined in the sense of eq. (2.2) with $\alpha>0$ and $k \geq 0$.) Suppose that the orbits of $X$ are complete through all points $p \in \Sigma_{\text {ext }}$. Let $\left\langle\left\langle M_{\text {ext }}\right\rangle\right\rangle$ denote the domain of outer communications associated with $\Sigma_{\text {ext }}$ as defined below. If $\left\langle\left\langle M_{\text {ext }}\right\rangle\right\rangle$ is globally hyperbolic, then the orbits of $X$ through points $p \in\left\langle\left\langle M_{\text {ext }}\right\rangle\right\rangle$ are complete.

In view of the recent classification of orbits of Killing vector field in asymptotically flat space-times of [1] it is of interest to prove the equivalent of Theorem 1.2 for "stationary-rotating" Killing vectors $X$, as defined in [1] . In Theorem 3.1 below we prove that generalization.

\section{Definitions, proof of Theorem 1.1.}

Throughout this work all objects under consideration are assumed to be smooth. For a vector field $W$ we denote by $\phi_{t}[W]$ the (perhaps defined only locally) flow generated by $\mathrm{W}$. Consider a Killing vector field $X$ which is timelike for $p \in \Sigma_{\text {ext }}$. If the orbits $\gamma_{p}$ of $X$ are complete through points $p \in \Sigma_{\text {ext }}$, then we define the asymptotically flat four-end $M_{\text {ext }}$ by

$$
M_{\mathrm{ext}}=\cup_{t \in \mathbb{R}} \phi_{t}[X]\left(\Sigma_{\mathrm{ext}}\right),
$$

and the domain of outer communications $\left\langle\left\langle M_{\text {ext }}\right\rangle\right\rangle$ by

$$
\left\langle\left\langle M_{\text {ext }}\right\rangle\right\rangle=J^{-}\left(M_{\text {ext }}\right) \cap J^{+}\left(M_{\text {ext }}\right) .
$$

Let $R>0$ and let $\left(g_{i j}, K_{i j}\right)$ be initial data on $\Sigma_{\text {ext }} \equiv \Sigma_{R} \equiv \mathbb{R}^{3} \backslash B(R)$ satisfying

$$
g_{i j}-\delta_{i j}=O_{k}\left(r^{-\alpha}\right), \quad K_{i j}=O_{k-1}\left(r^{-1-\alpha}\right),
$$

with some $k \geq 1$ and some $0<\alpha<1$. A set $\left(\Sigma_{\text {ext }}, g_{i j}, K_{i j}\right)$ satisfying the above will be called an asymptotically flat three-end. Here a function $f$, 
defined on $\Sigma_{R}$, is said to be $O_{k}\left(r^{\beta}\right)$ if there exists a constant $C$ such that we have

$$
0 \leq i \leq k \quad\left|\partial^{i} f\right| \leq C r^{\beta-i} .
$$

We shall need the following result, which is a straightforward consequence [ of what has been proved in [14]:

Theorem 2.1 (Nomizu) Let $\left(M, g_{a b}\right)$ be a (connected) simply connected analytic pseudo-Riemannian manifold, and suppose that there exists a Killing vector field $Y$ defined on an open connected subset $\mathcal{O}$ of $M$. Then there exists a Killing vector field $\hat{Y}$ defined on $M$ which coincides with $Y$ on $\mathcal{O}$.

Let us pass to the proof of Theorem 1.1. Without loss of generality we may assume that $X$ is future oriented for $p \in \Sigma_{\text {ext }}$. Simple connectedness and analyticity of $\left\langle\left\langle M_{\text {ext }}\right\rangle\right\rangle$ together with Theorem 2.1 allow us to conclude that the Killing vector $Y$ can be globally extended to a Killing vector field $\hat{Y}$ defined on $\left\langle\left\langle M_{\text {ext }}\right\rangle\right\rangle$. The time-likeness of the ADM four-momentum $p^{\mu}$ allows us to use the results in [1] to assert that there exists a linear combination $Z$ (with constant coefficients) of $X$ and $\hat{Y}$ which has complete periodic orbits through all points $p$ in $M_{\text {ext }}$ which satisfy $r(p) \geq R$, for some $R$. (Moreover $Z$ and $X$ commute.) To prove Theorem 1.1 we need to show that the orbits of $Z$ are complete (and periodic) for all $p \in\left\langle\left\langle M_{\text {ext }}\right\rangle\right\rangle$.

Consider, thus, a point $p \in\left\langle\left\langle M_{\text {ext }}\right\rangle\right\rangle$. There exist $q_{ \pm} \in M_{\text {ext }}$, with $r\left(q_{ \pm}\right) \geq$ $R$, such that $p \in J^{-}\left(q_{+}\right) \cap J^{+}\left(q_{-}\right)$. Completeness and periodicity of the orbits $\gamma_{q_{ \pm}}[Z] \equiv \cup_{t \in \mathbb{R}} \phi_{t}[Z]\left(q_{ \pm}\right)$of $Z$ through $q_{ \pm}$implies that the sets $\gamma_{q_{ \pm}}[Z]$ are compact. Global hyperbolicity of $\left\langle\left\langle M_{\text {ext }}\right\rangle\right\rangle$ implies then that

$$
K \equiv J^{-}\left(\gamma_{q_{+}}[Z]\right) \cap J^{+}\left(\gamma_{q_{-}}[Z]\right)
$$

is compact.

For $q \in\left\langle\left\langle M_{\text {ext }}\right\rangle\right\rangle$ let $t_{ \pm}(q) \in \mathbb{R} \cup\{ \pm \infty\}$ be the forward and backward life time of orbits of $Z$ through $q$, defined by the requirement that $\left(t_{-}(q), t_{+}(q)\right)$ is the largest connected interval containing 0 such that the solution $\phi_{t}[Z](q)$ of the equation $d \phi_{t}[Z](q) / d t=\circ \phi_{t}[Z](q)$ is defined for all $t \in\left(t_{-}(q), t_{+}(q)\right)$. From continuous dependence of solutions of ODE's upon initial values it

\footnotetext{
${ }^{6}$ Actually in 14$]$ it is assumed that $\left(M, g_{a b}\right)$ is Riemannian. The reader will note that all the assertions and proofs of [14] remain valid word for word when "Riemannian" is replaced by "pseudo-Riemannian".
} 
follows that $t_{+}$is a lower semi-continuous function and $t_{-}$is an upper semicontinuous function.

Let $\gamma:[0,1] \rightarrow M$ be any future oriented causal curve such that $\gamma(0)=$ $q_{-}, \gamma(1)=q_{+}$, and $p \in \gamma$. Set

$$
T_{+}=\inf _{q \in \gamma} t_{+}(q), \quad T_{-}=\sup _{q \in \gamma} t_{-}(q) .
$$

Here and elsewhere inf and sup are taken in $\mathbb{R} \cup\{ \pm \infty\}$. If $T_{ \pm}= \pm \infty$ we are done, suppose thus that $T_{+} \neq \infty$; the case $T_{-} \neq-\infty$ is analyzed in a similar way. By lower semi-continuity of $t_{+}$and compactness of $\gamma$ there exists $\tilde{p} \in \gamma$ such that $t_{+}(\tilde{p})=T_{+}$. By global hyperbolicity the family of causal curves $\phi_{t}[Z](\gamma), t \in\left[0, T_{+}\right)$, accumulates at a causal curve $\tilde{\gamma} \subset K$. Consequently the orbit $\phi_{t}[Z](\tilde{p}), t \in\left[0, T_{+}\right)$, has an accumulation point in $K$. It follows that $\phi_{t}[Z](\tilde{p})$ can be extended beyond $T_{+}$, which gives a contradiction unless $T_{+}=\infty$, and the result follows.

\section{Proof of Theorem 1.2.}

Proof of Theorem 1.2: Without loss of generality we may suppose that $X$ is future oriented for $p \in \Sigma_{\text {ext }}$. Consider a point $p \in\left\langle\left\langle M_{\text {ext }}\right\rangle\right\rangle$, there exist $p_{ \pm} \in M_{\text {ext }}$ such that $p \in J^{+}\left(p_{-}\right) \cap J^{-}\left(p_{+}\right)$. Let $\Sigma$ be a Cauchy surface for $\left\langle\left\langle M_{\text {ext }}\right\rangle\right\rangle$, without loss of generality we may assume that $p_{-} \in I^{-}(\Sigma)$ and $p_{+} \in I^{+}(\Sigma)$. Let $t_{ \pm}$be defined as in the proof of Theorem 1.1, we have $t_{-}\left(p_{ \pm}\right)=-\infty, t_{+}\left(p_{ \pm}\right)=\infty$. Let $\gamma:[0,1] \rightarrow\left\langle\left\langle M_{\text {ext }}\right\rangle\right\rangle$ be any causal curve such that $\gamma(0)=p_{-}, \gamma(1)=p_{+}$, and $p \in \gamma$. Define $T_{ \pm}$by eq. (2.3). By lower semi-continuity of $t_{+}$there exists $\tilde{p} \in \gamma$ such that $t_{+}(\tilde{p})=T_{+}$. Define

$$
\tilde{\Omega}=\left\{s \in\left[0, T_{+}\right): \phi_{s}[X](\tilde{p}) \in I^{-}(\Sigma)\right\} .
$$

Consider any $s \in \tilde{\Omega}$. Then the curve obtained by concatenating $\phi_{t}[X]\left(p_{-}\right)$, $t \in[0, s]$, with $\phi_{s}[X](\gamma)$ is a future directed causal curve which starts at $p_{-}$ and passes through $\phi_{s}[X](\tilde{p})$, hence

$$
s \in \tilde{\Omega} \Rightarrow \phi_{s}[X](\tilde{p}) \in K \equiv J^{+}\left(p_{-}\right) \cap J^{-}(\Sigma) .
$$

By global hyperbolicity of $\left\langle\left\langle M_{\text {ext }}\right\rangle\right\rangle$ the set $K$ is compact. If $\tilde{\Omega}=\emptyset$ set $\omega=0$, otherwise set

$$
\omega=\sup \tilde{\Omega} .
$$


Consider any sequence $\omega_{i} \in \tilde{\Omega}$ such that $\omega_{i} \rightarrow \omega$. By (3.1) and by compactness of $K$ the sequence $\phi_{\omega_{i}}[X](\tilde{p})$ has an accumulation point in $K$. It follows that $\omega<T_{+}$.

By definition of $\omega$ we have $\phi_{s}[X](\tilde{p}) \in J^{+}(\Sigma)$ for all $\omega \leq s<T_{+}$. By Lemma 2.5 of [5] it follows that $T_{+}=\infty$. As $t_{+}(p) \geq t_{+}(\tilde{p})=T_{+}$we obtain $t_{+}(p)=\infty$. The equality $t_{-}(p)=-\infty$ for all $p \in\left\langle\left\langle M_{\text {ext }}\right\rangle\right\rangle$ is obtained similarly by using the time-dual version of Lemma 2.5 of [5].

Before presenting a generalization of Theorem 1.2 which covers the case of "stationary-rotating" Killing vectors, as defined in 9, 1], we need to introduce some terminology. Following [9] we shall say that the orbit through $p$ of a Killing vector field $Z$ is time-oriented if there exists $t_{p}>0$ such that $\phi_{t_{p}}[Z](p) \in I^{+}(p)$. It then follows that for all $\alpha \in R$ and all $z \in \mathbb{N}$ we have $\phi_{\alpha+z t_{p}}[Z](p) \in I^{+}\left(\phi_{\alpha}[Z](p)\right)$ : if $\gamma$ is a timelike curve from $p$ to $\phi_{t_{p}}[Z](p)$, one obtains a timelike curve from $\phi_{\alpha}[Z](p)$ to $\phi_{\alpha+z t_{p}}[Z](p)$ by concatenating $\phi_{\alpha}[Z](\gamma)$ with $\phi_{\alpha+t_{p}}[Z](\gamma)$ with $\phi_{\alpha+2 t_{p}}[Z](\gamma)$, etc.

A trivial example of a Killing vector field with time-oriented orbits is given by a timelike Killing vector field. A more interesting example is that of "stationary-rotating" Killing vector fields, as considered in [9, 1] - loosely speaking, those are Killing vectors which behave like $\alpha \partial / \partial t+\beta \partial / \partial \phi$ in the asymptotic region, with $\alpha$ and $\beta$ non-vanishing, where $\phi$ is an angular coordinate. Thus the theorem that follows applies in the "stationary-rotating" case.

Theorem 3.1 The conclusion of Theorem 1.2 will hold if to its hypotheses one adds the requirement that $k$ in (2.8) is larger than or equal to 2, and if the hypothesis that $X$ is timelike is replaced by the assumption that the orbits of $X$ are time-oriented through all $p \in \Sigma_{\text {ext }}$.

Proof: The proof is achieved by a minor modification of the proof of Theorem 1.2, as follows: Let $p_{ \pm}$be as in that proof, from the asymptotic behavior of Killing vector fields in asymptotically flat space-times (cf. e.g. Section 2 of [2]) it follows that we can without loss of generality assume that

$$
\begin{gathered}
\phi_{2 \pi}[X]\left(p_{+}\right) \in I^{+}\left(p_{+}\right), \quad \phi_{2 \pi}[X]\left(p_{-}\right) \in I^{+}\left(p_{-}\right), \\
\forall s \in[0,2 \pi] \quad \phi_{s}[X]\left(p_{-}\right) \in I^{-}(\Sigma), \quad \phi_{s}[X]\left(p_{+}\right) \in I^{+}(\Sigma) .
\end{gathered}
$$

The proof proceeds then as before, up to the definition of the set $K$, eq. (3.1). In the present case that definition is replaced by

$$
K \equiv J^{+}\left(\cup_{s \in[0,2 \pi]} \phi_{s}[X]\left(p_{-}\right)\right) \cap J^{-}(\Sigma) .
$$


This set is again compact, in view of global hyperbolicity of $M_{\text {ext }}$. The fact that for $s \in \tilde{\Omega}$ we have $\phi_{s}[X](\tilde{p}) \in K$ follows by considering the causal curve obtained by concatenating a causal curve $\gamma_{1}$ from $\phi_{s-\lfloor s / 2 \pi\rfloor 2 \pi}[X](\tilde{p})$ to $\phi_{s}[X](\tilde{p})$ with $\phi_{s}[X](\gamma)$. Here $\lfloor\alpha\rfloor$ denotes the largest integer smaller than or equal to $\alpha$; the existence of $\gamma_{1}$ is guaranteed by our discussion above.

Acknowledgments: The author is grateful to I. Rácz for comments about a previous version of this paper.

\section{References}

[1] R. Beig and P.T. Chruściel, The isometry groups of asymptotically flat, asymptotically empty space-times with timelike ADM four-momentum, (1996), preprint.

[2] —, Killing vectors in asymptotically flat space-times: I. Asymptotically translational Killing vectors and the rigid positive energy theorem, Jour. Math. Phys. 37 (1996), 1939-1961, gr-qc/9510015.

[3] B. Carter, Black hole equilibrium states, Black Holes (C. de Witt and B. de Witt, eds.), Gordon \& Breach, New York, London, Paris, 1973, Proceedings of the Les Houches Summer School.

[4] P.T. Chruściel, On uniqueness in the large of solutions of Einstein equations ("Strong Cosmic Censorship"), Australian National University Press, Canberra, 1991.

[5] - On completeness of orbits of Killing vector fields, Class. Quantum Grav. 10 (1993), 2091-2101, gr-qc/9304029.

[6] —, "No Hair" Theorems - folklore, conjectures, results, Differential Geometry and Mathematical Physics (J. Beem and K.L. Duggal, eds.), vol. 170, American Mathematical Society, Providence, 1994, gr-qc/9402032, pp. 23-49.

[7] —, Uniqueness of black holes revisited, Univ. de Tours preprint, to appear in the Proceedings of Journées Relativistes, May 1996. 
[8] P.T. Chruściel and A. Rendall, Strong cosmic censorship in vacuum space-times with compact, locally homogeneous Cauchy surfaces, Annals of Phys. 242 (1995), 349-385.

[9] P.T. Chruściel and R.M. Wald, On the topology of stationary black holes, Class. Quantum Grav. 11 (1994), L147-152.

[10] G.J. Galloway, On the topology of the domain of outer communication, Class. Quantum Grav. 12 (1995), L99-L101.

[11] S.W. Hawking and G.F.R. Ellis, The large scale structure of space-time, Cambridge University Press, Cambridge, 1973.

[12] G.T. Horowitz, The positive energy theorem and its extensions, Asymptotic behavior of mass and spacetime geometry (F. Flaherty, ed.), Springer Lecture Notes in Physics, vol. 202, Springer Verlag, New York, 1984.

[13] T. Jacobson and S. Venkatarami, Topology of event horizons and topological censorship, Class. Quantum Grav. 12 (1995), 1055-1061.

[14] K. Nomizu, On local and global existence of Killing vector fields, Ann. Math. 72 (1960), 105-120. 